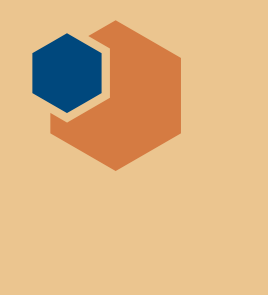

\section{NSF kicks off Quantum Leap Challenge Institutes program}

nsf.gov

M aterials research to advance quantum information science (QIS) has strong potential to revolutionize a range of technologies across sensing, computing, modeling, and communications. Development of these technologies will have a significant impact on the economy and national security of the United States, drawing interest from within the US government and driving QIS funding and policy (see sidebar). The latest action from the National Science Foundation (NSF) is a solicitation for funding within the Quantum Leap Challenge Institutes (QLCI) program. This is in addition to 18 awards announced for "conceptualization grants" already issued in 2019.

The conceptualization grants awarded under this program are a one-year investment of between $\$ 100,000$ and $\$ 150,000$ with the goal of supporting research teams as they develop Challenge Institute proposals. Starting dates for the conceptualization grants range from August 2019 to June 2020, and most are already underway. "The goal [of the conceptualization grant] is to form a compelling and comprehensive vision for a QLCI proposal," says Junichiro Kono, professor at Rice University and principal investigator for the Texas Quantum Institute, which is one of the awarded QLCI conceptualization grants.

The QLCI program solicitation runs through 2021 and will provide funding for between two and six Challenge Institutes funded at levels up to $\$ 5$ million per year for five years. Research at these institutes will focus on quantum sensing, computing, modeling, and/or communications.

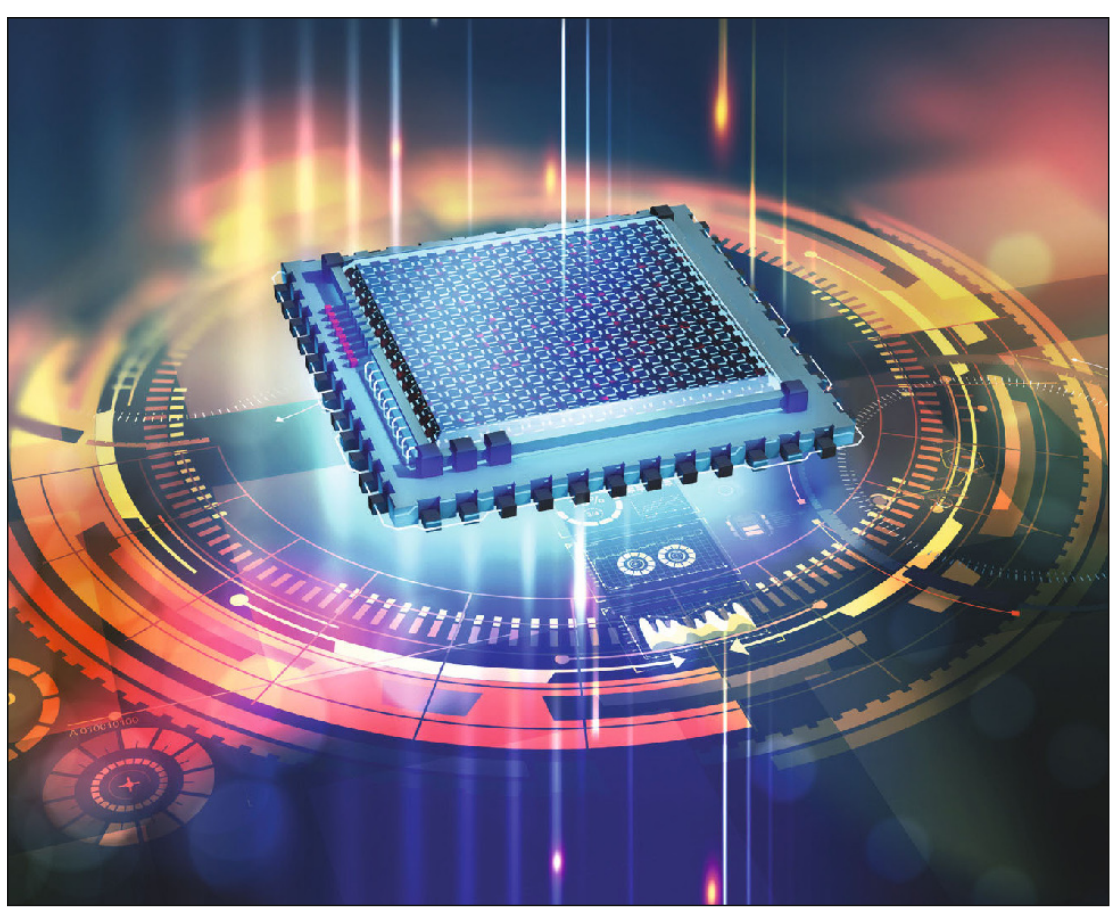

Three-dimensional conceptual illustration of quantum processor in a global computer network. Credit: Shutterstock.
In addition to addressing the scientific and technological challenges associated with quantum research, the institutes are tasked with finding "multidisciplinary approaches" to educate and build the future QIS workforce. The QLCI program satisfies statutory requirements laid out in the National Quantum Initiative Act mandating that NSF establish between two and five multidisciplinary QIS centers.

Kono says many of the researchers involved with building the Texas Quantum Institute are already working on QIS research. But Kono believes an additional value of the conceptualization grant is that it has enabled researchers to form new collaborations and find cross-disciplinary approaches to developing QIS technologies.

Kai-Mei Fu, principal investigator for the Institute for Hybrid Quantum Systems at the University of Washington in Seattle - another recipient of a QLCI conceptualization grant-agrees that while QIS research is already underway across a range of disciplines, there is a need to build connections across these disciplines to bring "people who were not formally trained as quantum information scientists to participate and contribute to the field of QIS." Fu, an associate professor of physics and electrical and computer engineering at the University of Washington, believes that these connections can be built through the quantum institutes, which will serve to consolidate knowledge, cultivate greater expertise, and leverage academic capabilities to accelerate development of QIS technologies.

Many of the groups that have received conceptualization grants have already held meetings or workshops to bring together team members, discuss challenges and opportunities in QIS research, and begin to map out a QLCI proposal. And while most of the conceptualization grants were awarded to named institutes, it is important to note that the quantum centers themselves do not yet exist - they will only become Challenge Institutes if they win a QLCI grant.

"By the end of the 12-month period, we will have built a well-defined network of team members with specific roles, identified compelling research themes 
for overcoming quantum challenges, and developed effective plans to attract and engage students and young researchers to produce next-generation researchers in quantum science and engineering," says Kono of their effort to secure a QLCI grant.

Both Kono and $\mathrm{Fu}$ recognize the important role of materials research in advancing quantum science, and both the Texas Quantum Institute and Institute for Hybrid Quantum Systems include materials researchers. Kono characterizes quantum materials as being an "essential underpinning” of future QIS technologies, and Fu says that "it is well understood that advancements in materials will need to be made to enable scalable quantum information technologies."

Producing materials of higher purity, increasing control over materials synthesis to produce desirable properties for quantum information, and developing new and more sensitive means of measuring materials properties are issues that, according to $\mathrm{Fu}$, must be addressed by materials scientists to enable the QIS technologies of the future. In addition,
$\mathrm{Fu}$ points to the potential use of quantum simulation to accelerate understanding and discovery of materials and says, "QIS and materials are profoundly related."

"This is a very exciting time for quantum information science," says Fu because not only has the US government recognized the importance of QIS, but it is also the "first time we are seeing a really significant investment into quantum technologies from industry." The institute structure that is part of the QLCI program will help further encourage industry investment and participation because it creates an environment where multidisciplinary teams from academia, government, and the private sector will need to come together to focus on large-scale challenges.
"A collection of individual investigators could never have the sustained, cross-disciplinary, holistic effort needed" to address the sizable challenges in both accelerating QIS advancements and developing the quantum information scientists and engineers of the future, Kono says. But Kono, Fu, and a host of other researchers are already utilizing the QLCI program to convene the teams and build the environment necessary to push the frontiers of QIS.

Jennifer A. Nekuda Malik

\section{EU companies increased R\&D investment in 2019}

$\mathrm{C}$ ompanies in the EU have increased their investment in R\&D for the ninth consecutive year, but levels remain below the global growth rate. In the 2018-2019 time frame, EU companies invested $4.7 \%$ more in R\&D. This growth is driven by the automobile, health, and information and communication technology (ICT) sectors.

In the face of an ever-increasing global technological race, the top 2500 industrial players worldwide increased their R\&D investment by $8.9 \%$ in 2018-2019. EU companies account for 17 of the top 50 corporate R\&D investors. The growth in investment by EU-based companies is, however, outpaced by their US and Chinese counterparts: the former increased their $\mathrm{R} \& \mathrm{D}$ investment in that time frame by $10.3 \%$, the latter by $26.7 \%$.
These are some of the main findings of the 2019 EU Industrial R\&D Investment Scoreboard (doi:10.2760/04570), published by the European Commission in December of last year. It provides an annual in-depth analysis of the most recent investment trends of the worlds' leading industrial R\&D players contributing to understanding the positioning of the EU companies in the global landscape.

Mariya Gabriel, EU Commissioner for Innovation, Research, Culture, Education, and Youth, says, "Companies based in the EU keep increasing their R\&D investments, but much more is needed in the global race for technological leadership in deep-tech and sustainability. We will launch a fully fledged European Innovation Council to invest in high-risk companies creating new markets. If we want Europe to drive the transition to a climate-neutral economy, we need to redouble our efforts."

EU-based automotive companies have contributed most to R\&D growth in the EU and prepare for the future with a broad patent portfolio to reduce vehicle emissions (behind Japan) and for autonomous vehicles. With aims to meet the UN sustainable development goals (SDGs), the report published, "Toyota had the most green patents but the top 25 global companies by number of green patents comprise EU firms such as Bosch, Volkswagen, Airbus and Rolls-Royce." Furthermore, to meet the SDGs, industrial R\&D contributed to technologies such as artificial intelligence, biotechnology, nanotechnology, graphene, and improved clean energy generation and storage technologies.

The EU Industrial R\&D Investment Scoreboard has been published annually since 2004 by the European Commission's Directorate-General for Research and Innovation and the Joint Research Centre. 